\title{
Consultation on UTUC, Stockholm 2018 aspects of diagnosis of upper tract urothelial carcinoma
}

\author{
Grzegorz Fojecki ${ }^{1}$. Anders Magnusson ${ }^{2}$. Olivier Traxer ${ }^{3}$. Joyce Baard ${ }^{4}$ Palle Jörn Sloth Osther ${ }^{5}$. Georg Jaremko ${ }^{6}$. \\ Christian Seitz ${ }^{7} \cdot$ Thomas Knoll $^{8} \cdot$ Guido Giusti $^{9} \cdot$ Marianne Brehmer $^{10}$ (1)
}

Received: 9 January 2019 / Accepted: 13 March 2019 / Published online: 26 March 2019

(C) The Author(s) 2019

\begin{abstract}
Purpose To summarize knowledge on upper urinary tract carcinoma (UTUC) regarding diagnostic procedures, risk factors and prognostic markers.

Methods A scoping review approach was applied to search literature in Pubmed, Web of Science, and Embase. Consensus was reached through discussions at Consultation on UTUC in Stockholm, September 2018.

Results Tumor stage and grade are the most important prognostic factors. CT urography (CTU) including corticomedullary phase is the preferred imaging modality. A clear tumor on CTU in combination with high-grade UTUC in urine cytology identifies high-risk UTUC, and in some cases indirect staging can be obtained. Bladder urine cytology has limited sensitivity, and in most cases ureterorenoscopy (URS) with in situ samples for cytology and histopathology are mandatory for exact diagnosis. Image-enhancing techniques, Image S1 and narrow-band imaging, may improve tumor detection at URS. Direct confocal laser endomicroscopy may help to define grade during URS. There is strong correlation between stage and grade, accordingly correct grading is crucial. The correlation is more pronounced using the 1999 WHO than the 2004 classification: however, the 1999 system risks greater interobserver variability. Using both systems is advisable. A number of tissue-based molecular markers have been studied. None has proven ready for use in clinical practice.

Conclusions Correct grading and staging of UTUC are mandatory for adequate treatment decisions. Optimal diagnostic workup should include CTU with corticomedullary phase, URS with in situ cytology and biopsies. Both WHO classification systems (1999 and 2004) should be used to decrease risk of undergrading or overtreatment.
\end{abstract}

Keywords Upper tract urothelial carcinoma $\cdot$ UTUC $\cdot$ Diagnostics $\cdot$ CT urography $\cdot$ Ureteroscopy $\cdot$ Diagnostic samples

Marianne Brehmer

marianne.brehmer@ki.se

1 Department of Urology, Hospital of Southern Jutland, Sønderborg, Denmark

2 Department of Radiology, University Hospital, Uppsala, Sweden

3 Hôpital Tenon, Sorbonne Université, Paris, France

4 Department of Urology, Amsterdam UMC, University of Amsterdam, Amsterdam, Holland

5 Urological Research Center, Lillebaelt Hospital, University of Southern Denmark, Vejle, Denmark
6 Department of Clinical Pathology and Cytology, Karolinska University Hospital Solna, Stockholm, Sweden

7 Department of Urology, Medical University of Vienna, Vienna, Austria

8 Department of Urology, Teaching Hospital University Tuebingen, Sindelfingen, Germany

9 Department of Urology, IRCCS San Raffaele Hospital, Ville Turro Division, Milan, Italy

10 Division of Urology, Department of Clinical Sciences, Danderyd Hospital, Karolinska Institutet, Stockholm, Sweden 


\section{Introduction}

Upper tract urothelial carcinoma (UTUC) has an incidence of less than two cases per 100000 in the Western world [1]. About $60 \%$ of tumors are invasive at the time of diagnosis [2]. Radical nephroureterectomy (RNU) was the standard treatment of UTUC but the European Association of Urology (EAU) guidelines in 2018 recommend that kidney-sparing surgery should be discussed in cases of low-risk UTUC. The EAU guidelines define low-risk UTUC according to these criteria, all of which must be fulfilled: unifocal disease, tumor size $<2 \mathrm{~cm}$, low-grade cytology and histology (biopsy), and no invasive aspects on computed tomography urography (CTU). High-risk indicators are any of the following: hydronephrosis, tumor size $>2 \mathrm{~cm}$, high-grade cytology and histology (biopsy), multifocality, previous radical cystectomy for bladder cancer, and variant histology. The risk factors are based mainly on retrospective reports, and the level of evidence is low. The EAU guidelines use the 2004 WHO classification system for grading tumors [3].

Grade and stage are the strongest prognostic factors $[4,5]$, whereas tumor size and multifocality may be less significant for the disease-specific survival [6]. Accordingly, correct grading and staging are highly important. The EAU guidelines for diagnostic workup include cystoscopy, CTU, ureterorenoscopy (URS) and biopsy in cases in which additional information can have an impact on treatment decisions [3].

This review aims to consider the challenges in achieving correct diagnosis of UTUC by discussing the different steps in diagnostic procedures, expanding the current recommendations, and showing directions for future research. A scoping review approach was applied to search literature in Pubmed, Web of Science, and Embase.

\section{Diagnostic workup}

\section{Radiological workup}

CTU has higher sensitivity than intravenous urography for diagnosing UTUC [7]. In patients with contraindication for use of contrast medium, magnetic resonance urography can be an option.

Studies have underlined the importance of the manner in which CTU is performed [8, 9]. Helenius et al. [10] showed that enhancement of urothelial carcinomas was highest in the corticomedullary phase (CMP) and, in a subsequent investigation [11] confirmed that, compared to other phases, the CMP offered superior sensitivity and negative predictive value for detection of urothelial cell carcinoma. Metser et al. [12] concluded that the urothelial phase was better than the excretory phase for detecting UTUC. In a prospective study, Grahn et al. [13] observed that multiphase CTU including a CMP offered $85 \%$ sensitivity in detecting UTUC. The sensitivity dropped to $58 \%$ when a non-optimized CT was performed. When comparing sensitivity and specificity of ureterorenoscopy (URS) and multiphase CT in UTUC, Grahn and co-workers found evidence that multiphase CTU might even provide higher sensitivity than endoscopy, although the specificity was significantly higher for URS. Tumors the researchers described as being difficult to visually diagnose at URS were carcinoma in situ (CIS), which has also been reported by other authors [14].

Current radiological methods cannot diagnose invasiveness unless the tumor is advanced. However, for staging, CT scan is informative regarding lymph node involvement or distant metastasis.

In conclusion, CTU including CMP is the preferred imaging modality in the diagnostic workup of UTUC (Fig. 1).

\section{Ureterorenoscopy}

The emergence of thinner instruments for flexible URS (fURS) with digital technology has improved the diagnostic value of URS. Using image enhancement techniques during URS can improve detection of urothelial tumors.

The Image $1 \mathrm{~S}^{\mathrm{TM}}$ system (Karl Storz Endoscopy) modifies white-light (WL) images into four different imaging modalities designated as Spectra A, Spectra B, Chroma, and Clara. The Spectra A modality highlights the contrast of capillaries and vessels in the mucosa, Spectra B modality reduces the red spectral reflection highlighting the contrast between tissues and structures, Chroma enhances the sharpness, and Clara enhances local brightness adaptation in the image with clearer visibility of darker regions. The modes can be activated simultaneously in a combined Clara \& Chroma mode $(\mathrm{C} \& \mathrm{CH})$. Kamphuis et al. [15] compared the performance of $\mathrm{C} \& \mathrm{CH}$ with that of WL cystoscopy, investigating 80 bladder tumors, and concluded that $\mathrm{C} \& \mathrm{CH}$ and Spectra B offered the best tumor visualization. Emiliani et al. [16] used standardized templates to assess image quality with regard to color contrast and image definition of Flex XC (Karl Storz, Tüttlingen, Germany). Clara and $\mathrm{C} \& \mathrm{CH}$ scored better than WL ( $p=0.0139$ and $p<0.05$, respectively), but Spectra A and Spectra B scored worse than WL $(p=0.0005$ and $p=0.0023$ ). Using the same standardized template, Talso et al. [17] compared seven different fURS systems (Lithovue, Olympus V, Olympus V2, Storz Flex XC, Wolf Cobra vision, Olympus P6, and Storz Flex X2) and found that digital scopes offered better image quality than fiber 
Fig. 1 Mutiphase CT urography of a tumor in the right renal pelvis. In the excretion phase (a, b), a filling defect is clearly visible in both the axial and the coronal plane, although this defect may have been caused by a blood clot. In the corticomedullary phase (c, d), the lesion is contrast enhanced, proving that there is tumor growth
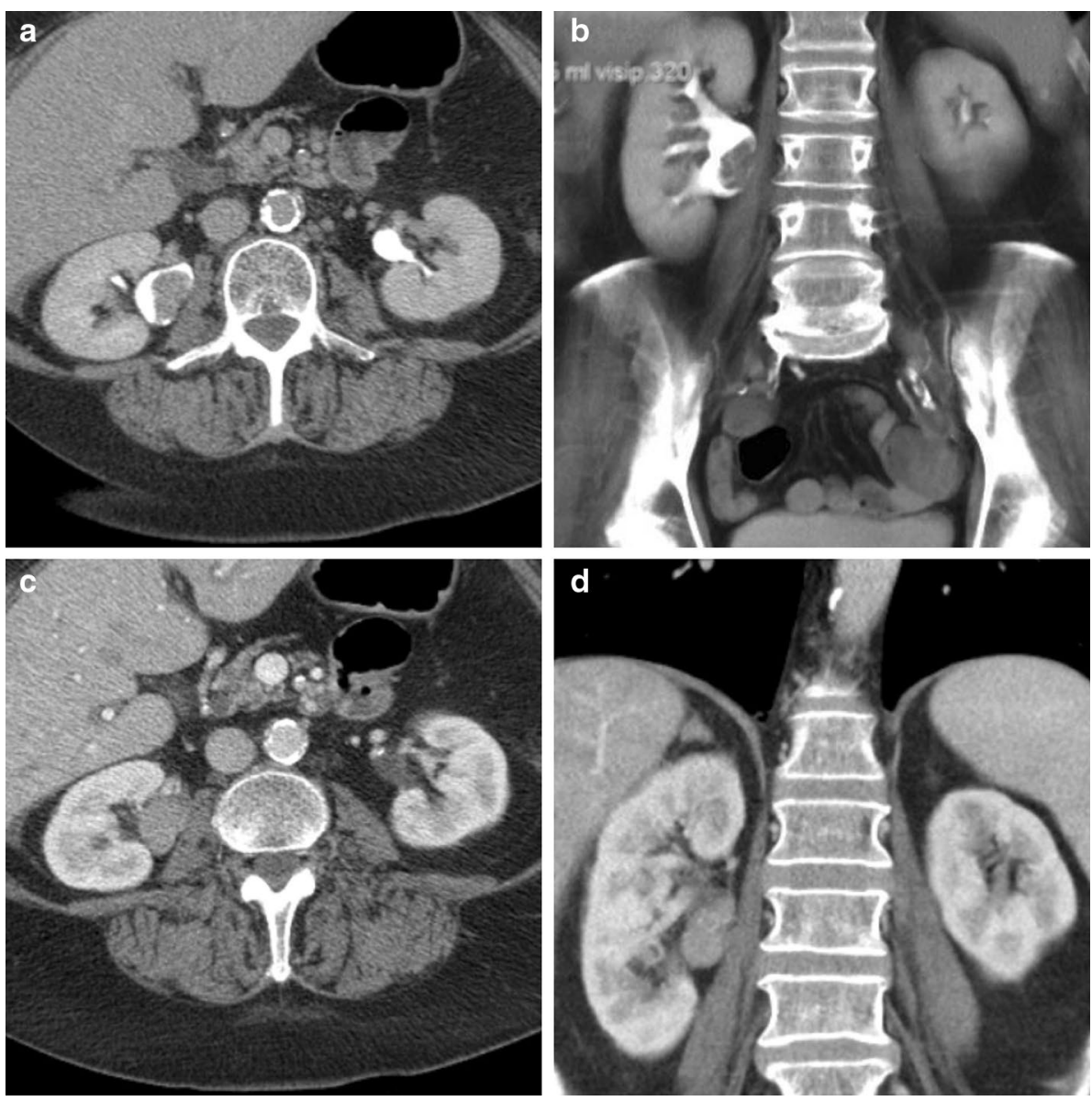

optics. The best overall performance was provided by Flex XC with $\mathrm{C} \& \mathrm{CH}(p<0.0001)$. Clinical studies documenting the value of Image $1 \mathrm{~S}$ technology are needed.

Narrow-band imaging (NBI) (Olympus Surgical, Orangeburg, NY) is an optical image enhancement technology narrowing the wavelength to 415 and $540 \mathrm{~nm}$, corresponding to blue and green light, respectively. This specific light is absorbed by hemoglobin, thereby increasing the visibility of capillaries at different depths. A meta-analysis (including $>1000$ patients) demonstrated that, compared with WL, NBI achieved up to $24 \%$ higher detection of bladder tumors [18] and offered greater sensitivity both per person and per lesion [19].

Two prospective studies have evaluated NBI for detection of UTUC. Traxer et al. [20] performed URS on 27 patients with UTUC, applying WL and NBI; Compared with WL, NBI detected five additional tumors and also revealed extended tumor growth in three cases. Hao et al. [21] obtained nearly identical results in 54 fURS examinations performed on 16 patients. The studies show that NBI may enhance visualization of tumors and potentially improve both diagnostics and treatment of UTUC.

\section{Biopsy for histopathology}

Tumor grade and stage are key factors in risk stratification of UTUC. Therefore, biopsy for histopathology is an important part of the diagnostic procedure. There is no consensus regarding which biopsy method is preferable to achieve representative samples [22]. Harvesting good-quality tissue samples was accomplished in only $75 \%$ of cases in a study by Tavora et al [23]. In a consecutive cohort of patients who underwent RNU at three centers in the United States in 2000-2016, initial biopsy, using cold cup forceps or basket, underestimated tumor grade in $18 \%$ of cases [24]. There was significant discordance between URS biopsy and final pathology for UTUC: positive predictive values (PPVs) for invasiveness of high grade on biopsy was $60 \%$; negative predictive values (NPVs) for invasiveness of low grade on biopsy was $80 \%$. Smith et al. [25] documented even greater discrepancy between biopsy and final pathology of RNU specimens: $43 \%$ (24/56) of the tumors were upgraded after RNU. Limitations of the biopsy technique and tumor heterogeneity are possible explanations of initial underestimation of grade and stage [26]. 
Margolin et al. [24] noted that the likelihood of missing invasion on URS biopsy was significantly increased when the diameter of biopsy fragments was $\leq 1 \mathrm{~mm}$ (OR 4.3). To overcome this limitation, larger biopsy forceps (10 Fr cup), BIGopsy (COOK Medical, USA), have been developed. Three studies [27-29] compared BIGopsy forceps with standard biopsy techniques and nitinol baskets, and concluded that BIGopsy retrieved biopsies more appropriate for histopathological evaluation. However, they also identified disadvantages of BIGopsy, including decreased visibility and the necessity of using ureteral access sheaths (UASs) for backloading into the endoscope. Basket biopsy is excellent for exophytic tumors, whereas cup forceps are most suitable for flat lesions [28, 29].

Although biopsy quality may be improved by a larger biopsy device or a multi-biopsy approach [30], it has been suggested that addition of cytology should be mandatory to improve grading [24]. Messer et al. [31] showed that positive urinary cytology had sensitivity of $56 \%$ and a PPV of $54 \%$ for high-grade UTUC, and corresponding values of $62 \%$ and $44 \%$ for muscle-invasive UTUC; using selective upper tract cytology increased the PPV for detecting high-grade lesions to $85 \%$. In a meta-analysis of the diagnostic utility of selective upper tract urinary cytology [32], pooled sensitivity of selective cytology with respect to final pathology was $53.1 \%$, and corresponding values for low- and high-grade tumors were $45.6 \%$ and $69.9 \%$, respectively. Thus, selective cytology alone is not perfect for grading and staging UTUC. In a prospective study of 51 patients, Keeley et al. [33] used both biopsy and cytology. Fresh samples for cytology were taken in 48 subjects, whereas biopsy material was sufficient in 42 of those cases. This approach increased the concordance rate with final pathology to $90 \%$ for low-moderate-grade lesions and to $91.6 \%$ for high-grade lesions. Importantly, there was significant correlation between grade and stage in this study. Williams et al. [34] obtained similar results when using a nearly identical protocol in a retrospective analysis.

Similarly, in a study of a prospective series of UTUC patients $(n=43)$, Malm et al. [35] used a strict protocol comprising bladder barbotage before manipulation, nontouch URS, renal pelvis barbotage, and collection of fluid in the bladder after URS to detect ureteral tumors. With this approach, Malm and colleagues showed that cytology was as effective as final pathology to identify malignancy and to assess grade. Hence, it seems that strict sampling protocols including cytopathological evaluation of fresh samples may increase sensitivity for both grading and staging.

\section{Confocal laser endomicroscopy}

Confocal laser endomicroscopy (CLE) enables real-time visualization of tissue microarchitecture and cellular morphology. A fluorescent contrast agent that stains extracellular matrix of the tissue is applied in the upper urinary tract and a mobile laser $(488 \mathrm{~nm})$ scanning unit with a fiber optic probe is used to scan the tissue. In the urinary tract, CLE has mainly been used to distinguish between low- and highgrade lesions in the bladder [36]. Three reports have discussed the feasibility of applying CLE in the upper urinary tract [37-39] for grading of UTUC. All three investigations were based on small case series but nonetheless show high concordance with low-grade UTUC and CIS on final pathology. Most of the studied CLE specimens were compared with biopsy samples and not with RNU specimens, limiting the validity of the observations. The technique does seem promising but must be further evaluated.

\section{Grading and staging}

Grade and stage are the strongest prognostic factors in UTUC [3-5]. Direct staging of biopsies is difficult, since samples seldom contain tumor base with lamina propria [22]. However, there is a strong correlation between stage and grade $[3,40]$. When using the $2004 \mathrm{WHO}$ classification, low-grade tumors are more likely to be regarded as superficial, whereas it is likely that high-grade tumors will be identified as invasive. The stage-grade correlation seems to be greater for UTUC than for bladder cancer. With the 1973/1999 WHO system, grading tumors as G1-G3, the correlation is very strong for G1 (superficial) and G3 (invasive) tumors, but is more unpredictable for $\mathrm{G} 2$ lesions [4, 22, 34]. Brown et al. [41] conducted a retrospective study of 184 UTUC patients undergoing RNU and found that $100 \%, 71.7 \%$, and $33.8 \%$ of G1, G2, and G3 tumors, respectively, were superficial. Maruschke et al. [7] reported similar results, although a larger proportion (88\%) of the G3 tumors was invasive. Holmäng and Johansson [42] recut and re-evaluated 555 UTUC specimens from patients with no previous or concomitant bladder cancer. They concluded that the 1973/1999 WHO classification is superior to the $2004 \mathrm{WHO}$ system for clinical use since the correlation between stage and grade was strong when using the 1973/1999 WHO system, but was poor with the 2004 WHO classification. Using the 1973/1999 WHO system, the correlation with stage was strong for G1 and G3 tumors, but was less pronounced for G2 tumors, which may be important when deciding treatment modality [5]. In addition, the disease-specific survival differed between G1 and G2 tumors with the 1973/1999 WHO, an observation that was difficult to assess with the 2004 WHO system.

\section{Histopathological considerations}

The 1973 and 1999 WHO systems for grading of urothelial tumors have been the most extensively used and widely accepted approaches [43]. However, more detailed grading also entails the risk of greater interobserver variability. G2 
is most difficult to assess, which has led to the ambiguous use of diagnostic categories such as "grade 1-2" and "grade 2-3". There has also been confusion regarding G2 (pTa) tumors in that such cases can include high-risk disease. These issues led to introduction of the 1998 WHO/ISUP classification, later revised and presented as the 2004 and the 2016 WHO systems. The three-tiered 1999 WHO classification was divided into a low- and a high-grade category with the intention of ensuring that most patients with highrisk disease would be sorted into the high-grade category (Fig. 2). The morphological criteria were clearly detailed in an earlier study reported by Malmström et al. [44].

The rationale for using a classification system including only a low and a high grade is supported by the existence of two distinct molecular pathways of neoplastic transformation in urothelial tumors in the bladder [45]. Lowgrade tumors have a large proportion of FGFR3 alterations $(80 \%)$. They have a high recurrence rate but non-aggressive behavior. High-grade tumors have an elevated frequency of TP53. They are associated with a high risk of progression to muscle-invasive disease. The 2004 classification offers the advantage of greater interobserver reproducibility [46]. The EAU recommends that the new system is used together with the WHO 1999 grading classification, because it allows comparison of long-term outcomes at different clinical centers.

\section{Cytology}

The Paris system for reporting urinary cytology has gained broad acceptance [47]. It includes the following diagnostic categories:

1. Non-diagnostic/unsatisfactory

2. Negative for high-grade urothelial carcinoma

3. Atypical urothelial cells

4. Suspicious for high-grade urothelial carcinoma

5. High-grade urothelial carcinoma

6. Low-grade urothelial neoplasm
7. Other: primary and secondary malignancies and miscellaneous lesions

The main objective is to identify high-grade UTUC including all G3 and some G2 tumors. "Atypical urothelial cells" is used only when high-grade urothelial carcinoma is suspected, i.e., when there is an indication of aggressive cancer.

\section{Molecular markers for UTUC}

The difficulties in correct grading of UTUC based on the cytology and biopsies indicate the need for more reliable tumor markers.

In a retrospective study of 83 tumors, Bagrodia et al. [48] observed that only two alterations were uniformly related to high-grade and advanced disease: TP53/MDM2 alterations, associated with poor prognosis; and FGFR3 mutations, associated with more favorable outcome. In another study, Bagrodia et al. [49] also found high concordance in genomic alterations between tumor biopsies and subsequent RNU specimens.

Although many different tissue markers have been investigated, none of them have shown the potential to change clinical practice. Most studies have examined gene malformation in RNU specimens, often within selected patient groups. Published investigations of tissue markers are summarized in Table 1.

\section{Potential risks of URS in UTUC}

The risks associated with URS in treatment of UTUC can be subdivided into two groups: [1] general risks, including high intrarenal pressure (IRP) and ureteral damage caused by the use of a UAS; and [2] specific risks, including issues related to delaying final therapy and tumor seeding. High IRP resulting in tubular and pyelovenous backflow during URS is the main cause of septic and haemorrhagic complications, as well as access-related complications, because IRP induces peristalsis that can compromise access [50].

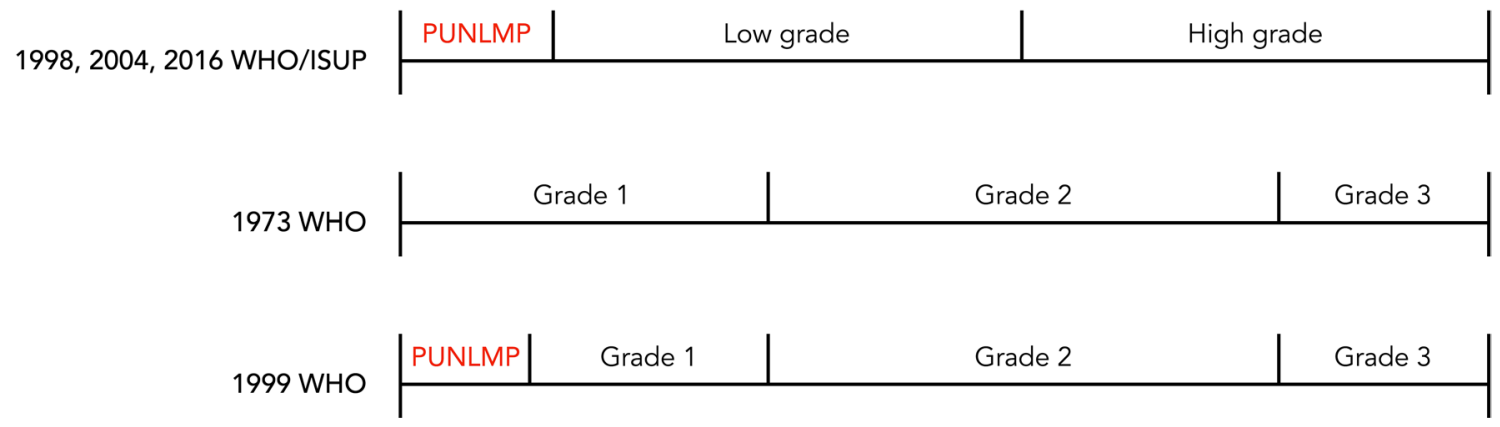

Fig. 2 WHO classification systems for grading of urothelial tumors. PUNLMP papillary urothelial neoplasm with low malignant potential 
Table 1 Studies of molecular markers for detection of UTUC

\begin{tabular}{|c|c|c|c|c|}
\hline Tissue marker & Year & Study type & No. of patients & Conclusion \\
\hline Gene malformations [47] & 2017 & Retrospective & 83 & $\begin{array}{l}\text { TP53/MDM2 pathway alterations and FGFR3 mutations associ- } \\
\text { ated with high-grade; FGFR3 associated with more favorable } \\
\text { outcome }\end{array}$ \\
\hline Gene malformations [48] & 2018 & Retrospective & 39 & $\begin{array}{l}\text { TERT and FGFR } 3 \text { mutations observed in } 64 \% \text { of high-grade } \\
\text { tumors (ureteroscopic biopsies comparable to RNU speci- } \\
\text { mens) }\end{array}$ \\
\hline Ki-67 [49] & 2015 & Prospective, multicentre & 475 & Independent predictor of RFS and CSS \\
\hline HER-2 [50] & 2014 & Retrospective & 171 & $\begin{array}{l}\text { HER-2 gene overexpression was found in } 18.1 \% \text { of cases and in } \\
\text { multivariate analysis was correlated with early tumor recur- } \\
\text { rence in the bladder }\end{array}$ \\
\hline HER-2 [51] & 2017 & Retrospective, multicentre & 732 & $\begin{array}{l}\text { Multivariate analysis confirmed that HER- } 2 \text { overexpression was } \\
\text { associated with RFS and CSS, }\end{array}$ \\
\hline EGFR, Ki-67 [52] & 2016 & Retrospective & 320 & $\begin{array}{l}\text { An independent risk factor for bladder cancer recurrence after } \\
\text { RNU }\end{array}$ \\
\hline
\end{tabular}

$R F S$ recurrence-free survival, CSS cancer-specific survival, HER-2 human epidermal growth factor receptor type 2, EGFR epidermal growth factor receptor, $R N U$ radical nephroureterectomy

High IRP can result in forniceal rupture and potentially also perirenal tumor seeding [50]. Such seeding has been reported to occur along a percutaneous nephrostomy tract in UTUC patients [51], although no studies in the literature have concerned perirenal or intratubular seeding following URS. Nevertheless, it is crucial to maintain low IRP during URS in UTUC to reduce both general and specific risks related to the disease. Prospective data on UAS usage in UTUC are lacking. Gorin et al. [52] have described findings suggesting that UAS can facilitate acquisition of high-quality specimens. This information was compiled in an assessment of a retrospective cohort representing 85 procedures with UAS application performed on 64 patients. Sufficient material was obtained in $90.4 \%$ of the cases, and concordance with final pathology was found in $88.6 \%$, and the authors concluded that UAS application was safe in UTUC. However, Lildal et al. [53] showed that ureteral trauma following UAS application is often underestimated. It is even possible that injuries to the muscular coat of the ureter can be missed. Such lesions can serve as nidi for through-the-mucosa tumor cell seeding. Thus, it is still a matter of debate whether use of a UAS during URS in UTUC should be recommended. Data must be compiled regarding the safety of UAS usage in UTUC management.

The potential risk of intravesical recurrence after diagnostic URS is well known, although this aspect has not been found to have an impact on overall, cancer-specific, recurrence-free, or metastasis-free survival $[54,55]$. Furthermore, Maruschke et al. [7] and Chitale et al. [56]. studied RNUs that were performed based on radiographic findings and without prior URS. Their results showed that in $5.6 \%(6 / 113)$ and $10.2 \%(4 / 39)$ of cases, respectively, no tumor was found on final pathology, highlighting the considerable risk of overtreatment if URS is not performed in patients with suspected UTUC.

\section{Conclusions}

Diagnostic workup of patients with suspected UTUC comprises the following: CTU, preferably with a CMP, for detection of tumors as contrast-enhancing masses rather than as filling defects; cystoscopy and urinary cytology; fURS with biopsy and renal pelvis barbotage cytology.

URS image enhancement systems, Image $1 \mathrm{~S}$ and NBI, may improve both diagnosis and outcome of local treatment. General risks of URS must be taken into consideration. It appears that URS does not affect the final oncological outcome in UTUC patients, although this procedure does seem to be associated with an increased risk of intravesical recurrence.

There is a strong correlation between tumor grade and stage, and accordingly grading is crucial. The use of strict sampling protocols including cytopathological evaluation of fresh specimens increases the sensitivity of both grading and staging. Both of the WHO classification systems (1999 and 2004) should be used for grading to decrease the risk of undergrading or overtreatment.

Molecular markers for predicting aggressive UTUC are warranted. Further studies in this field are needed.

Acknowledgements We are grateful to Boston Scientific, Cook Medical, Apsu Medical, Olympus, Karl Storz Endoscope, Micron Med, Rocamed and Medac for supporting the Stockholm Consultation on UTUC meeting. We thank chief assistant Katrine Lindholtz for outstanding administrative management and Patricia Ödman for excellent language revision. 
Author contributions GF: manuscript writing. AM: data collection and analysis, presentation, editing of manuscript. OT: data collection and analysis, presentation, editing of manuscript. JB: data collection and analysis, presentation, editing of manuscript. GJ: data collection and analysis, presentation, editing of manuscript. CS: data collection and analysis, presentation, editing of manuscript. TK: analysis of data and editing of manuscript. GG: analysis of data and editing of manuscript. PO: project co-ordination, collection and analysis of data, presentation, writing and editing of manuscript. $\mathrm{MB}^{*}$ : project co-ordination, collection and analysis of data, presentation, writing and editing of manuscript.

\section{Compliance with ethical standards}

Conflict of interest Grzegorz Fojecki: no conflict of interest. Anders Magnusson: no conflict of interest. Olivier Traxer: Consultant for Coloplast, Boston Scientific, Olympus, Rocamed, EMS and IPG medical. Joyce Baard: Consultant for BSC and Coloplast, Speaker honoraria from Karl Storz Endoscope, Olympus and Cook Medical, Financial research support from Astellas and GSK. Georg Jaremko: no conflict of interest. Christian Seitz: no conflict of interest. Thomas Knoll: no conflict of interest. Guido Giust: Consultant for Olympus, Boston Scientific, Rocamed, Cook Medical and Puanta System. Palle Osther: no conflict of interest. Marianne Brehmer*: no conflict of interest.

Informed consent Informed consent is not relevant.

Human and animal rights This is a review article involving no studies on human participants and/or animals.

Open Access This article is distributed under the terms of the Creative Commons Attribution 4.0 International License (http://creativeco mmons.org/licenses/by/4.0/), which permits unrestricted use, distribution, and reproduction in any medium, provided you give appropriate credit to the original author(s) and the source, provide a link to the Creative Commons license, and indicate if changes were made.

\section{References}

1. Siegel RL, Miller KD, Jemal A (2017) Cancer statistics, 2017. CA Cancer J Clin 67(1):7-30

2. Margulis V et al (2009) Outcomes of radical nephroureterectomy: a series from the Upper Tract Urothelial Carcinoma Collaboration. Cancer 115(6): 1224-1233

3. Rouprêt M, Babjuk M, Burger M, Compérat E, Cowan NC, Gontero P, Mostafid AH, Palou J, van Rhijn BWG, Shariat SF, Sylvester R, Zigeuner R (2018) EAU guidelines on upper urinary tract urothelial carcinoma. Eur Urol 73(1):111-122

4. Elawdy MM et al (2016) Histopathologic characteristics of upper tract urothelial carcinoma with an emphasis on their effect on cancer survival: a single-institute experience with 305 patients with long-term follow-up. Clin Genitourin Cancer 14(6):e609-e615

5. Holmäng S, Johansson SL (2005) Urothelial carcinoma of the upper urinary tract: comparison between the WHO/ISUP 1998 consensus classification and WHO 1999 classification system. Urology 66(2):274-278

6. Villa L et al (2018) Which patients with upper tract urothelial carcinoma can be safely treated with flexible ureteroscopy with holmium: YAG laser photoablation? Long-term results from a high volume institution. J Urol 199(1):66-73
7. Maruschke $\mathrm{M}$ et al (2017) Upper urinary tract tumors: which diagnostic methods are needed? Urol Int 98(3):304-311

8. Takeuchi M et al (2015) CT urography for diagnosis of upper urinary tract urothelial carcinoma: are both nephrographic and excretory phases necessary? Am J Roentgenol 205(3):W320-W327

9. Gandrup KL et al (2014) Upper urinary tract tumors: how does the contrast enhancement measured in a split-bolus CTU correlate to histological staging? Acta Radiol 55(6):761-768

10. Helenius $M$ et al (2014) Contrast enhancement in bladder tumors examined with CT urography using traditional scan phases. Acta Radiol 55(9):1129-1136

11. Helenius $M$ et al (2016) Comparison of post contrast CT urography phases in bladder cancer detection. Eur Radiol 26(2):585-591

12. Metser U et al (2012) Detection of urothelial tumors: comparison of urothelial phase with excretory phase CT urography-a prospective study. Radiology 264(1):110-118

13. Grahn A et al (2016) Diagnostic accuracy of computed tomography urography and visual assessment during ureterorenoscopy in upper tract urothelial carcinoma. BJU Int 119(2):289-297

14. Gillan A et al (2015) Carcinoma in situ is significantly underdetected by prenephroureterectomy ureteroscopy in the management of upper tract urothelial cancers. Biomed Res Int 2015:547586

15. Kamphuis GM et al (2016) Comparing image perception of bladder tumors in four different Storz professional image enhancement system modalities using the iSPIES App. J Endourol 30(5):602-608

16. Emiliani E et al (2017) Evaluation of the Spies (TM) modalities image quality. Int Braz J Urol 43(3):476-480

17. Talso $\mathrm{M}$ et al (2018) Comparison of flexible ureterorenoscope quality of vision: an in vitro study. J Endourol 32(6):523-528

18. Li K et al (2012) Diagnosis of narrow-band imaging in nonmuscle-invasive bladder cancer: a systematic review and metaanalysis. Int J Urol 20(6):602-609

19. Zheng $C$ et al (2012) Narrow band imaging diagnosis of bladder cancer: systematic review and meta-analysis. BJU Int 110(11b):E680-E687

20. Traxer O et al (2011) Narrow-band imaging digital flexible ureteroscopy in detection of upper urinary tract transitional-cell carcinoma: initial experience. J Endourol 25(1):19-23

21. Hao YC et al (2018) Application of narrow-band imaging flexible ureteroscopy in the diagnosis, treatment and follow-up of upper tract urothelial carcinomas. Chin J Surg 56(3):222

22. Skolarikos A et al (2003) Cytologic analysis of ureteral washings is informative in patients with grade 2 upper tract TCC considering endoscopic treatment. Urology 61(6):1146-1150

23. Tavora F et al (2009) Small endoscopic biopsies of the ureter and renal pelvis: pathologic pitfalls. Am J Surg Pathol 33(10):1540-1546

24. Margolin EJ et al (2018) Discordance between ureteroscopic biopsy and final pathology for upper tract urothelial carcinoma. J Urol 199(6):1440-1445

25. Smith AK et al (2011) Inadequacy of biopsy for diagnosis of upper tract urothelial carcinoma: implications for conservative management. Urology 78(1):82-86

26. Hayashi $\mathrm{H}$ et al (2017) Variant morphology in upper urinary tract urothelial carcinoma: a 14-year case series of biopsy and resection specimens. Hum Pathol 65:209-216

27. Al-Qahtani SM et al (2014) Can we improve the biopsy quality of upper urinary tract urothelial tumors? Single-center preliminary results of a new biopsy forceps. Urol Int 93(1):34-37

28. Lama DJ et al (2018) Multi-institutional evaluation of upper urinary tract biopsy using backloaded cup biopsy forceps, a Nitinol basket, and standard cup biopsy forceps. Urology 117:89-94 
29. Breda A, Territo A, Sanguedolce F, Basile G, Subiela JD, Reyes HV, Ferrer OM, Gaya JM, Palou J (2018) Comparison of biopsy devices in upper tract urothelial carcinoma. World J Urol. https:// doi.org/10.1007/s00345-018-2586-y

30. Guarnizo E et al (2000) Ureteroscopic biopsy of upper tract urothelial carcinoma: improved diagnostic accuracy and histopathological considerations using a multi-biopsy approach. J Urol 163(1):52-55

31. Messer J et al (2011) Urinary cytology has a poor performance for predicting invasive or high-grade upper-tract urothelial carcinoma. BJU Int 108(5):701-705

32. Potretzke AM et al (2016) Diagnostic utility of selective upper tract urinary cytology: a systematic review and meta-analysis of the literature. Urology 96:35-43

33. Keeley FX et al (1997) Diagnostic accuracy of ureteroscopic biopsy in upper tract transitional cell carcinoma. J Urol 157(1):33-37

34. Williams SK et al (2008) Correlation of upper-tract cytology, retrograde pyelography, ureteroscopic appearance, and ureteroscopic biopsy with histologic examination of upper-tract transitional cell carcinoma. J Endourol 22(1):71-76

35. Malm C et al (2017) Diagnostic accuracy of upper tract urothelial carcinoma: how samples are collected matters. Scand J Urol 51(2):137-145

36. Wu K et al (2011) Dynamic real-time microscopy of the urinary tract using confocal laser endomicroscopy. Urology 78(1):225-231

37. Breda A, Territo A, Guttilla A, Sanguedolce F, Manfredi M, Quaresima L, Gaya JM, Algaba F, Palou J, Villavicencio H (2017) Correlation between confocal laser endomicroscopy (Cellvizio ${ }^{\circledR}$ ) and histological grading of upper tract urothelial carcinoma: a step forward for a better selection of patients suitable for conservative management. Eur Urol Focus 4(6):954-959. https://doi. org/10.1016/j.euf.2017.05.008

38. Villa L et al (2015) Confocal laser endomicroscopy in the management of endoscopically treated upper urinary tract transitional cell carcinoma: preliminary data. J Endourol 30(2):237-242

39. Bui D et al (2015) A pilot study of in vivo confocal laser endomicroscopy of upper tract urothelial carcinoma. J Endourol 29(12):1418-1423

40. Brien JC et al (2010) Preoperative hydronephrosis, ureteroscopic biopsy grade and urinary cytology can improve prediction of advanced upper tract urothelial carcinoma. J Urol 184(1):69-73

41. Brown GA et al (2007) Ability of clinical grade to predict final pathologic stage in upper urinary tract transitional cell carcinoma: implications for therapy. Urology 70(2):252-256

42. Holmäng S, Johansson SL (2014) Long-term follow-up of patients with tumours of the renal pelvis and ureter: how often is a bladder tumour diagnosed after five tumour-free years? Scand J Urol 48(1):65-72

43. Mostofi FK, Sobin LH, Torloni H (1973) Histological typing of urinary bladder tumours. International histological classification of tumours, No. 10. World Health Organisation, Geneva
44. Malmstrom PU, Busch BJ, Fau-Norlen C, Norlen BJ (1987) Recurrence, progression and survival in bladder cancer. A retrospective analysis of 232 patients with greater than or equal to 5-year follow-up. Scand J Urol Nephrol 21:185-195

45. Wu X-R (2005) Urothelial tumorigenesis: a tale of divergent pathways. Nat Rev Cancer 5(9):713-725

46. Epstein JIMDA, Mahul BMD, Reuter Victor R MD, Mostofi Fathollah K MD (1998) The World Health Organization/International Society of Urological Pathology consensus classification of urothelial (transitional cell) neoplasms of the urinary bladder. Am J Surg Pathol 22(12):1435-1448

47. Barkan GA et al (2016) The Paris system for reporting urinary cytology: the quest to develop a standardized terminology. Acta Cytol 60(3): 185-197

48. Bagrodia A et al (2016) Genomic biomarkers for the prediction of stage and prognosis of upper tract urothelial carcinoma. J Urol 195(6):1684-1689

49. Bagrodia A et al (2018) Genomic profile of urothelial carcinoma of the upper tract from ureteroscopic biopsy: feasibility and validation using matched radical nephroureterectomy specimens. Eur Urol Focus. https://doi.org/10.1016/j.euf.2018.01.005

50. Osther PJS (2018) Risks of flexible ureterorenoscopy: pathophysiology and prevention. Urolithiasis 46(1):59-67

51. Schwartzmann I et al (2017) Upper urinary tract urothelial carcinoma tumor seeding along percutaneous nephrostomy track: case report and review of the literature. Urol Int 98(1):115-119

52. Gorin MA et al (2011) Initial clinical experience with use of ureteral access sheaths in the diagnosis and treatment of upper tract urothelial carcinoma. Urology 78(3):523-527

53. Lildal SK et al (2017) Histopathological correlations to ureteral lesions visualized during ureteroscopy. World J Urol 35(10):1489-1496

54. Guo R-Q et al (2017) Impact of ureteroscopy before radical nephroureterectomy for upper tract urothelial carcinomas on oncological outcomes: a meta-analysis. BJU Int 121(2):184-193

55. Marchioni $\mathrm{M}$ et al (2017) Impact of diagnostic ureteroscopy on intravesical recurrence in patients undergoing radical nephroureterectomy for upper tract urothelial cancer: a systematic review and meta-analysis. BJU Int 120(3):313-319

56. Chitale $\mathrm{S}$ et al (2008) Nephroureterectomy for transitional cell carcinoma - the value of pre-operative histology. Ann R Coll Surg Eng1 90(1):45-50

Publisher's Note Springer Nature remains neutral with regard to jurisdictional claims in published maps and institutional affiliations. 